\section{No cumplir con las recomendaciones de actividad física se asocia a mayores niveles de obesidad, diabetes, hipertensión y síndrome metabólico en población chilena}

\author{
XIMENA DÍAZ-MARTÍNEZ ${ }^{1, a^{*}}$, FANNY PETERMANN $2, b^{*}$, \\ ANA MARÍA LEIVA', , ALEX GARRIDO-MÉNDEZ ${ }^{4, \mathrm{~d}}$, \\ CARLOS SALAS-BRAVO ${ }^{5, a}$, MARÍA ADELA MARTÍNEZ ${ }^{6, e}$, \\ ANA MARÍA LABRAÑA ${ }^{7, \mathrm{f}}$, ELIANA DURAN ${ }^{7, g}$, \\ PEDRO VALDIVIA-MORAL ${ }^{8, \mathrm{~d}}$, MARÍA LUISA ZAGALAZ ${ }^{9, \mathrm{~d}}$, \\ FELIPE POBLETE-VALDERRAMA ${ }^{10, \mathrm{~h}}$, CRISTIAN ALVAREZ ${ }^{11, \mathrm{i}}$, \\ CARLOS CELIS-MORALES ${ }^{2,12, j}$; EN REPRESENTACIÓN DEL GRUPO \\ DE INVESTIGACIÓN ELHOC (EPIDEMIOLOGY OF LIFESTYLEAND \\ HEALTH OUTMCOMES IN CHILE)

\section{Association of physical inactivity with obesity, diabetes, hypertension and metabolic syndrome in the chilean population}

Background: Physical inactivity is an important cardiovascular risk factor. Aim: To investigate the association of physical inactivity with obesity, metabolic markers, type 2 diabetes mellitus, hypertension and metabolic syndrome in Chilean adults. Material and Methods: Participants from the National Health Survey 2009-10 $(n=5,157)$ were included in this study. Body mass index, waist circumference, metabolic markers (blood glucose, glycosylated hemoglobin and lipid profile) were the outcomes. Type 2 diabetes, hypertension and metabolic syndrome were determined using international criteria. Physical activity levels were determined using the Global Physical Activity Questionnaire and physical inactivity was defined as < 600 METs/minutes/week. Results: Compared to their physically active peers, inactive men and women had a higher odds ratio (OR) for obesity (OR: 1.77 [95\% confidence intervals (CI): 1.29-2.42], $p<0.01$ and 1.25 [95\% CI: 102-1.54], $p<0.035$, respectively), diabetes (OR: 2.47 [1.80-3.38], $p<0.01$ and 1.72 [1.35-2.19], $p=0.002$, respectively) and hypertension (OR: 1.66 [1.31-2.09], $p<0.01$ and 1.83 [1.54-2.18] respectively. An association of physical inactivity with central obesity and metabolic syndrome was observed only in men (OR: 1.92 [1.42- 2.58], $p<0.01$ and 1.74 [1.23-2.47], $p<0.01$, respectively). Conclusions: Not meeting the physical activity recommendations is associated with obesity, diabetes, hypertension and metabolic syndrome, which are important cardiovascular risk factors.

(Rev Med Chile 2018; 146: 585-595)

Key words: Cardiovascular Diseases; Diabetes Mellitus, Type 2; Metabolic Syndrome; Obesity; Sedentary Lifestyle.
'Grupo de Investigación Calidad de Vida Departamento Ciencias de la Educación, Universidad del Biobío, Chillán, Chile. ${ }^{2}$ BHF Glasgow Cardiovascular Research Centre, Institute of Cardiovascular and Medical Science, University of Glasgow, Glasgow, United Kingdom.

${ }^{3}$ Instituto de Anatomía, Histología y Patología. Facultad de Medicina, Universidad Austral de Chile. Valdivia. Chile.

${ }^{4}$ Departamento de Ciencias del Deporte y Acondicionamiento Físico. Universidad Católica de la Santísima Concepción.

Chile.

${ }^{5}$ Departamento de Educación Física. Facultad de Educación. Universidad de Concepción. Concepción, Chile. ${ }^{6}$ Instituto de Farmacia, Facultad de Ciencias, Universidad Austral de Chile. Valdivia, Chile.

${ }^{7}$ Departamento de Nutrición y Dietética y Programa de Magíster en Nutrición Humana, Facultad de Farmacia, Universidad de Concepción. Concepción, Chile.

${ }^{8}$ Universidad de Granada (España). Grupo de Investigación del PAIDI, HUM653, Innovación Didáctica en Actividad Física (IDAF).

${ }^{9}$ Universidad de Jaén (España). PI Grupo de Investigación del PAIDI, HUM653, Innovación Didáctica en Actividad Física (IDAF).

${ }^{10}$ Escuela de Kinesiología, Facultad de Salud, Universidad Santo Tomás, Sede Valdivia. Valdivia, Chile.

${ }^{11}$ Núcleo de Investigación en Salud, Actividad Física y Deporte, Universidad de Los Lagos, Osorno, Chile.

${ }^{12}$ Centro de Investigación en Fisiología del Ejercicio - CIFE, Universidad Mayor, Santiago, Chile.

aprofesor de Educación Física, MSc en Educación Física.

butricionista. MSc Nutrición Humana.

'Profesora de Biología y Química. MSc.

Neurociencias y Salud Mental.

dProfesor de Educación Física, Doctores en

Educación Física y en Educación.

eBioquímica. MSc. Nutrición y Dietética.

${ }^{f}$ Nutricionista. MSc. Ciencias de la

Educación.

${ }^{9}$ Nutricionista. MSc. Planificación en

Alimentación y Nutrición.

hProfesor de Educación Física, MSc. Educación en Salud y Bienestar Humano. iProfesor Educación Física, MSc. en entrenamiento deportivo. Doctor en

Ciencias de la Salud.

jprofesor de Educación Física, Doctor en Ciencias Cardiovasculares y Biomédicas.

*XDM y FP contribuyeron de igual

forma a este manuscrito por ende son consideradas primeras autoras compartidas.

Los autores declaran no tener conflictos de interés.

Trabajo no recibió financiamiento. Recibido el 31 de diciembre de 2016, aceptado el 18 de abril de 2018 .

Correspondencia a:

Dr. Carlos Celis-Morales BHF Glasgow Cardiovascular Research Centre. 126 University Avenue. Glasgow University. Glasgow. United Kingdom. G12 8TA

carlos.celis@glasgow.ac.uk 
L a inactividad física se ha convertido en uno de los factores de riesgo más importante en el desarrollo de enfermedades crónicas no transmisibles (ECNTs) a nivel mundial ${ }^{1-3}$. Ser inactivo $(<150 \mathrm{~min}$ de sus niveles de actividad física (AF) de intensidad moderada o vigorosa a la semana o su equivalente a realizar $<600$ Metabolic-energy-equivalents [METs]/minuto/semana) es causante de $6 \%$ a $10 \%$ del riesgo de sufrir enfermedad coronaria, hipertensión arterial (HTA), diabetes mellitus tipo 2 (DMT2), cáncer de mama y de colon ${ }^{1}$. Se estima que $9 \%$ de las muertes prematuras están asociadas con la inactividad física, esto equivale a 5,3 millones de muerte por año ${ }^{1}$. Por otra parte, a nivel económico, el costo asociado a la inactividad física fue de 53,8 billones de dólares en el año 2013 a nivel mundial ${ }^{4}$, mientras que se estima que el costo total asociado a no cumplir con las recomendaciones de AF para el sistema de salud en Chile es de US\$ 103 millones de dólares al año ${ }^{4}$.

A pesar de que existe suficiente evidencia científica que confirma los beneficios de la práctica regular de $\mathrm{AF}^{3,5-10}$, actualmente $31,1 \%$ de la población adulta a nivel mundial no cumple con las recomendaciones mínimas de AF. En Chile $19,8 \%$ de la población reportó ser físicamente inactiva según la encuesta nacional de salud (ENS) $2009-2010^{11,12}$. Si bien la práctica de AF se asocia a una reducción en el riesgo de desarrollar obesidad, DMT2, HTA y síndrome metabólico en población internacional ${ }^{8,13-16}$, se desconoce si cumplir con la recomendación de AF se asocia a una disminución en el riesgo de desarrollar estas condiciones. En consecuencia, el objetivo de este estudio fue investigar la asociación entre inactividad física con obesidad, marcadores de salud metabólicos, DMT2, HTA y síndrome metabólico en población chilena.

\section{Materiales y Métodos}

\section{Diseño del estudio}

La muestra seleccionada comprende a los participantes de la Encuesta Nacional de Salud desarrollada entre octubre del año 2009 y septiembre del año 2010 (ENS 2009-2010) ${ }^{11}$. La ENS 2009-2010 corresponde a un estudio de prevalencia realizado en hogares en una muestra nacional, probabilística, estratificada y multietápica de 5.412 personas mayores de 15 años con representa- tividad nacional, regional, y área urbana/rural. Un total de 5.157 participantes con información disponible en relación a sus niveles de AF fueron incluidos en este estudio. A pesar de que el resto de los participantes ( $5 \%$ del total) no fueron seleccionados, contaban con características similares a los seleccionados.

El protocolo de estudio fue aprobado por el Comité de Ética de Investigación de la Escuela de Medicina de la Pontificia Universidad Católica de Chile, todos los participantes firmaron un consentimiento informado ${ }^{11}$.

\section{Mediciones antropométricas y metabólicas}

Las mediciones antropométricas fueron realizadas por personal entrenado y han sido descritas en extenso en otra publicación ${ }^{11}$. El peso corporal y talla fueron utilizados para determinar el Îndice de Masa Corporal (IMC: $\mathrm{Kg} / \mathrm{m}^{2}$ ). Se clasificó el estado nutricional en base a las recomendaciones de la Organización Mundial de Salud (OMS) (bajo peso: IMC $<18,5 \mathrm{Kg} / \mathrm{m}^{2}$; normal: $\geq 18,5 \mathrm{y}<24,9$ $\mathrm{Kg} / \mathrm{m}^{2}$; sobrepeso: $\geq 25,0 \mathrm{y}<30,0 \mathrm{Kg} / \mathrm{m}^{2}$; obesidad $\left.\geq 30,0 \mathrm{Kg} / \mathrm{m}^{2}\right)^{17}$. El perímetro de cintura (PC) fue medido en la línea axilar media, en el punto medio entre reborde costal y cresta ilíaca, con una cinta métrica en centímetros. Para definir obesidad central se usaron los puntos de corte del Programa de Salud Cardiovascular de Chile ${ }^{17}: \geq 90 \mathrm{~cm} \mathrm{y} \geq 80$ $\mathrm{cm}$ en hombres y mujeres, respectivamente. Las muestras de sangre de los participantes fueron obtenidas en ayuno por una enfermera entrenada siguiendo protocolos estandarizados a nivel nacional ${ }^{11}$. Los marcadores metabólicos de glicemia, hemoglobina glicosilada (Hbac1), perfil lipídico (triglicéridos, colesterol HDL, colesterol LDL y colesterol total) y presión arterial fueron medidos con métodos estandarizados y previamente descritos en la ENS ${ }^{11}$. Los puntos de corte utilizados para el diagnóstico de factores de riesgo cardiovascular fueron los siguientes: HTA (PAS $\geq 140$ o PAD $\geq 90$ $\mathrm{mm} \mathrm{Hg}$ ), DMT2 (glicemia en ayuno $\geq 126 \mathrm{mg} / \mathrm{dl}$ ). El síndrome metabólico fue determinado utilizando el criterio de la IDF-ATP ${ }^{11,18}$, que requiere al menos tres de los siguientes cinco criterios para su clasificación: presión arterial $(>130 / 85 \mathrm{~mm}$ $\mathrm{Hg}), \mathrm{PC}$ aumentado basado en población chilena ( $\geq 90 \mathrm{~cm}$ en hombres $\mathrm{o} \geq 80 \mathrm{~cm}$ en mujeres $)^{17}$, colesterol HDL $(<40 \mathrm{mg} / \mathrm{dl}$ en hombres o $<50$ en mujeres), glicemia elevada $(>100 \mathrm{mg} / \mathrm{dl})$ y triglicéridos elevados $(>150 \mathrm{mg} / \mathrm{dl})$. 
Las variables socio-demográficas (edad, sexo, nivel educacional, ingreso económico) y datos asociados con estilos de vida, como el tabaquismo, consumo de frutas, verduras y sal, se obtuvieron mediante la aplicación de cuestionarios validados en la ENS ${ }^{11}$.

\section{Clasificación de actividad física}

El tiempo destinado a AF relacionada al transporte (ej. caminar, andar en bicicleta), y actividades de intensidad moderada o vigorosa realizadas durante el tiempo libre y/o en el trabajo, fueron determinadas según la guía de análisis de GPAQ (Global Physical Activity Questionnaire v2 $)^{19}$. Para determinar los niveles de AF total, las variables fueron expresadas en METs. Se consideró como punto de corte para inactividad física un gasto energético $<600 \mathrm{METs} / \mathrm{min} /$ semana, o su equivalente de $150 \mathrm{~min}$ de $\mathrm{AF}$ de intensidad moderada o 75 min de AF de intensidad vigorosa a la semana o su combinación, según las recomendación de la OMS y especificaciones en la guía de análisis de $\mathrm{GPAQ}^{10,19}$. Los niveles de sedentarismo fueron determinados mediante el auto-reporte de tiempo destinado a actividades que involucren estar sentado o reclinado durante el tiempo libre o de trabajo (Ej. tiempo sentado frente al computador, viendo televisión, viajando en bus, tren o auto, etc.).

\section{Análisis estadístico}

Para todos los análisis se utilizó el módulo de muestras complejas del programa Stata SE v14 y todos los resultados fueron estimados utilizando muestras expandidas según la ENS 2009-2010 ${ }^{11}$. La asociación entre $\mathrm{AF}$ (físicamente activo e inactivo) y variables continuas, como peso corporal, IMC, PC y marcadores metabólicos, fue investigada mediante análisis de regresión lineal, ajustado por variables de confusión (edad, tabaquismo, nivel educacional, sedentarismo, consumo de frutas, verduras y sal).

La asociación entre inactividad física y las variables de salida o outcomes (obesidad, marcadores metabólicos, DMT2, HTA y síndrome metabólico) fue investigada mediante análisis de regresión logística, siendo el grupo físicamente activo utilizado como referencia para estos análisis. Todos los análisis fueron estratificados según sexo y ajustados por edad, tabaquismo, nivel educacional, sedentarismo, consumo de frutas, verduras y sal. Los análisis para marcadores metabólicos, DMT2, síndrome metabólico e HTA fueron ajustados adicionalmente por IMC. Los datos para estos análisis fueron presentados como promedio (para variables continuas u Odds ratio (OR) para variables categóricas y sus respectivos intervalos de confianza de $95 \%$ (95\% IC)). El nivel de significancia fue definido como $\mathrm{p}<0,05$.

\section{Resultados}

Las características de la población estudiada según niveles de AF son presentadas en la Tabla 1. En resumen, los participantes clasificados como físicamente inactivos son mayoritariamente mujeres, tienen mayor edad, nivel de escolaridad básica o media, mayor prevalencia de obesidad y obesidad central. En relación con el estilo de vida, se destaca que las personas físicamente inactivas destinan más tiempo a actividades de tipo sedente.

Las diferencias en niveles de AF según sexo son presentadas en la Figura 1. Estos resultados muestran que las mujeres realizan menos $\mathrm{AF}$ que los hombres en todas las intensidades (AF total, de transporte, moderada y vigorosa); Sin embargo, los hombres reportan mayor tiempo sedente que las mujeres.

En la Figura 2 se presentan las diferencias en las variables antropométricas según nivel de $\mathrm{AF}$ y sexo. Estos resultados revelan que los hombres que son físicamente inactivos presentan un mayor peso corporal, IMC y PC en comparación a aquellos que reportaron ser físicamente activos; No obstante, en mujeres estas diferencias fueron significativas solo para PC.

Las diferencias en marcadores de salud metabólica, según niveles de AF y sexo, son presentados en la Figura 3. Estos resultados revelan que tanto hombres como mujeres que son físicamente inactivos tiene mayor PAS, PAD y glicemia en comparación a individuos físicamente activos. También observamos que la concentración de Hbacl fue significativamente mayor en hombres físicamente inactivos en comparación a aquellos físicamente activos; sin embargo, la concentración de Hbacl no fue significativamente diferente según niveles de $\mathrm{AF}$ en mujeres. No se encontraron diferencias significativas en el perfil lipídico según niveles de AF.

Finalmente, en la Figura 4 se presenta la asociación entre inactividad física y obesidad, 
Tabla 1. Características de la población chilena según recomendación de actividad física

\begin{tabular}{|c|c|c|c|}
\hline Variables & $\begin{array}{c}\text { Físicamente activo } \\
\geq 600 \mathrm{METs} / \mathrm{min} / \mathrm{semana}\end{array}$ & $\begin{array}{c}\text { Físicamente inactivo } \\
<600 \mathrm{METs} / \mathrm{min} / \mathrm{semana}\end{array}$ & Valor $\mathbf{p}$ \\
\hline \multicolumn{4}{|l|}{ Socio-demográficas } \\
\hline $\mathrm{n}$ & 3.923 & 1.234 & - \\
\hline Mujeres, n (\%) & $2.247(57,3)$ & $826(66,9)$ & $<0,0001$ \\
\hline Edad (años [DE]) & $44,5(17,6)$ & $52,5(20,7)$ & $<0,0001$ \\
\hline \multicolumn{4}{|l|}{ Grupo etario, n (\%) } \\
\hline$<25$ años & $639(16,3)$ & $142(11,5)$ & \\
\hline 25-44 años & $1.358(34,6)$ & $327(26,5)$ & $<0,0001$ \\
\hline 45-64 años & $1.331(33,9)$ & $360(29,2)$ & \\
\hline$\geq 65$ años & $595(15,2)$ & $405(32,8)$ & \\
\hline \multicolumn{4}{|l|}{ Zona geográfica, n (\%) } \\
\hline Rural & $585(14,9)$ & $177(14,3)$ & \\
\hline Urbano & $3.336(85,1)$ & $1.057(85,7)$ & 0,619 \\
\hline \multicolumn{4}{|l|}{ Nivel educacional, n (\%) } \\
\hline Básica & $930(23,7)$ & $438(36,0)$ & \\
\hline Media & $2.246(57,3)$ & $559(45,9)$ & $<0,0001$ \\
\hline Técnico Universitaria & $744(19,0)$ & $220(18,1)$ & \\
\hline \multicolumn{4}{|l|}{ Nivel de ingreso, n (\%) } \\
\hline Bajo & $2.068(55,2)$ & $683(58,5)$ & \\
\hline Medio & $1.265(33,7)$ & $365(31,3)$ & 0,137 \\
\hline Alto & $416(11,1)$ & $120(10,2)$ & \\
\hline \multicolumn{4}{|l|}{ Antropométricas } \\
\hline \multicolumn{4}{|l|}{ Estado nutricional IMC, n (\%) } \\
\hline$<18,5 \mathrm{~kg} / \mathrm{m}^{2}$ & $63(1,8)$ & $14(1,3)$ & \\
\hline $18,5-24,9 \mathrm{~kg} / \mathrm{m}^{2}$ & $1.062(29,7)$ & $292(26,5)$ & $<0,0001$ \\
\hline $25,0-29,9 \mathrm{~kg} / \mathrm{m}^{2}$ & $1.475(41,2)$ & $411(37,3)$ & \\
\hline$\geq 30,0 \mathrm{~kg} / \mathrm{m}^{2}$ & $978(27,3)$ & $385(34,9)$ & \\
\hline Obesidad central, n (\%)* & $3.371(85,9)$ & $1.124(91,1)$ & $<0,0001$ \\
\hline \multicolumn{4}{|l|}{ Estilos de vida } \\
\hline Actividad física total (MET/h/semana [DE]) & $154,7(147)$ & $1,54 \quad(2,8)$ & $<0,0001$ \\
\hline Actividad física de transporte (min/día [DE]) & $63,4 \quad(92,4)$ & $2,79 \quad(5,7)$ & $<0,0001$ \\
\hline Actividad física moderada (min/día [DE]) & $136,3(157)$ & $0,29 \quad(1,9)$ & $<0,0001$ \\
\hline Actividad física vigorosa (min/día [DE]) & $65,9(135)$ & $0,11 \quad(0,9)$ & $<0,0001$ \\
\hline Tiempo sedente (h/día [DE]) & $3,12 \quad(2,42)$ & $4,38 \quad(3,09)$ & $<0,0001$ \\
\hline Consumo de frutas y vegetales (g/día [DE]) & $216,9(137,4)$ & $208,9(140,7)$ & 0,103 \\
\hline Consumo de sal (g/día [DE]) & $9,71 \quad(2,7)$ & $9,89 \quad(3,6)$ & 0,147 \\
\hline \multicolumn{4}{|l|}{ Tabaquismo, n (\%) } \\
\hline Nunca & $1.547(39,5)$ & $578(47,5)$ & \\
\hline Ex-fumador & $935(23,8)$ & $293(24,0)$ & $<0,0001$ \\
\hline Fumador & $1.441(36,7)$ & $347(28,5)$ & \\
\hline
\end{tabular}

Datos presentados como promedio y desviación estándar (DE) para variables continúas y como número de observaciones y sus respectivos \% para variables categóricas. Se consideró como punto de corte para inactividad física un gasto energético < 600 $\mathrm{METs} / \mathrm{min} / \mathrm{semana}$, según las recomendación de la OMS y especificaciones en la guía de análisis de GPAQ. *Obesidad central fue definida en base al Consenso MINSAL Chile 2014 Enfoque de riesgo para la prevención de enfermedades cardiovasculares que recomienda puntos de corte para obesidad abdominal $\geq 90 \mathrm{~cm}$ en hombres $y \geq 80 \mathrm{~cm}$ en mujeres. 


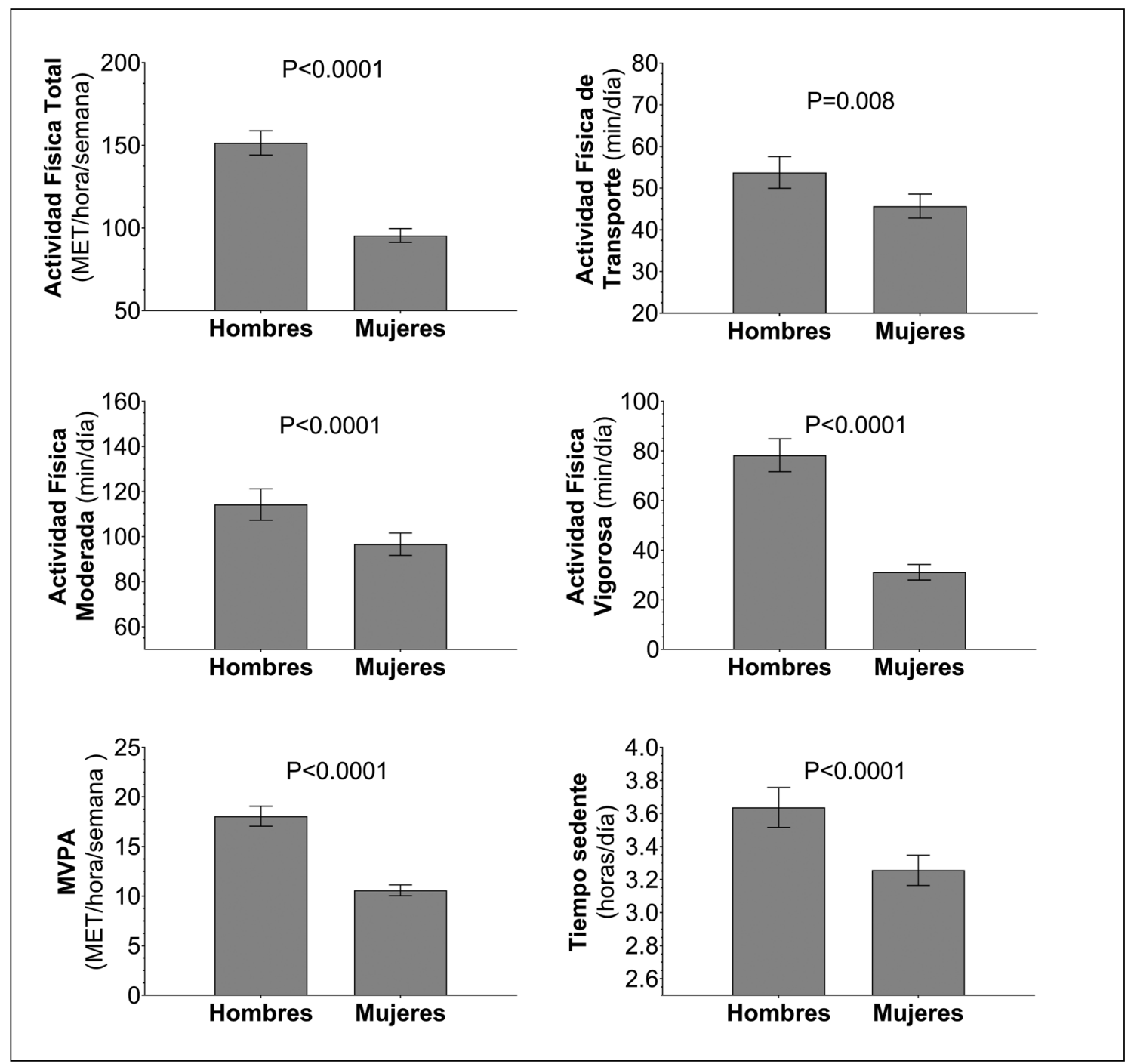

Figura 1. Niveles de actividad física y tiempo sedente según sexo en población chilena. Datos presentados como promedio y $95 \%$ IC. Los análisis fueron ajustados por edad, nivel de escolaridad y zona geográfica. Niveles de actividad física fueron determinados mediante el cuestionario GPAQ v2. MVPA (Actividad física de intensidad moderada a intensa).

DMT2, HTA y síndrome metabólico según sexo. Estos resultados revelan que el no cumplimiento de las recomendaciones de AF, se asocia a una mayor probabilidad de ser obeso (OR: 1,77 [95\% IC: 1,$29 ; 2,42$ ], $\mathrm{p}<0,0001$ y 1,25 [95\% IC: 102 ; $1,54], \mathrm{p}=0,035$ ), diabético (OR: 2,47 [1,80; $3,38], \mathrm{p}<0,0001$ y $1,72[1,35 ; 2,19], \mathrm{p}=0,002)$ e hipertenso (OR: 1,66 [1,31; 2,09], $\mathrm{p}<0,0001$ y $1,83[1,54 ; 2,18], p<0,0001)$, en hombres $y$ mujeres, respectivamente. Sin embargo, ser físi- camente inactivo se asocia a una mayor probabilidad de obesidad central (OR: $1,92[1,42 ; 2,58]$, $\mathrm{p}<0,0001)$ y síndrome metabólico (OR: 1,74 $[1,23 ; 2,47], \mathrm{p}<0,0001)$ en hombres pero no en mujeres (obesidad central OR: $1,01[0,73 ; 1,41]$, $\mathrm{p}=0,919$; síndrome metabólico OR: $1,18[0,90$; $1,54], p=0,217)$. Todos estos resultados fueron ajustados por edad, tabaquismo, nivel educacional, sedentarismo, consumo de frutas, verduras y sal. 


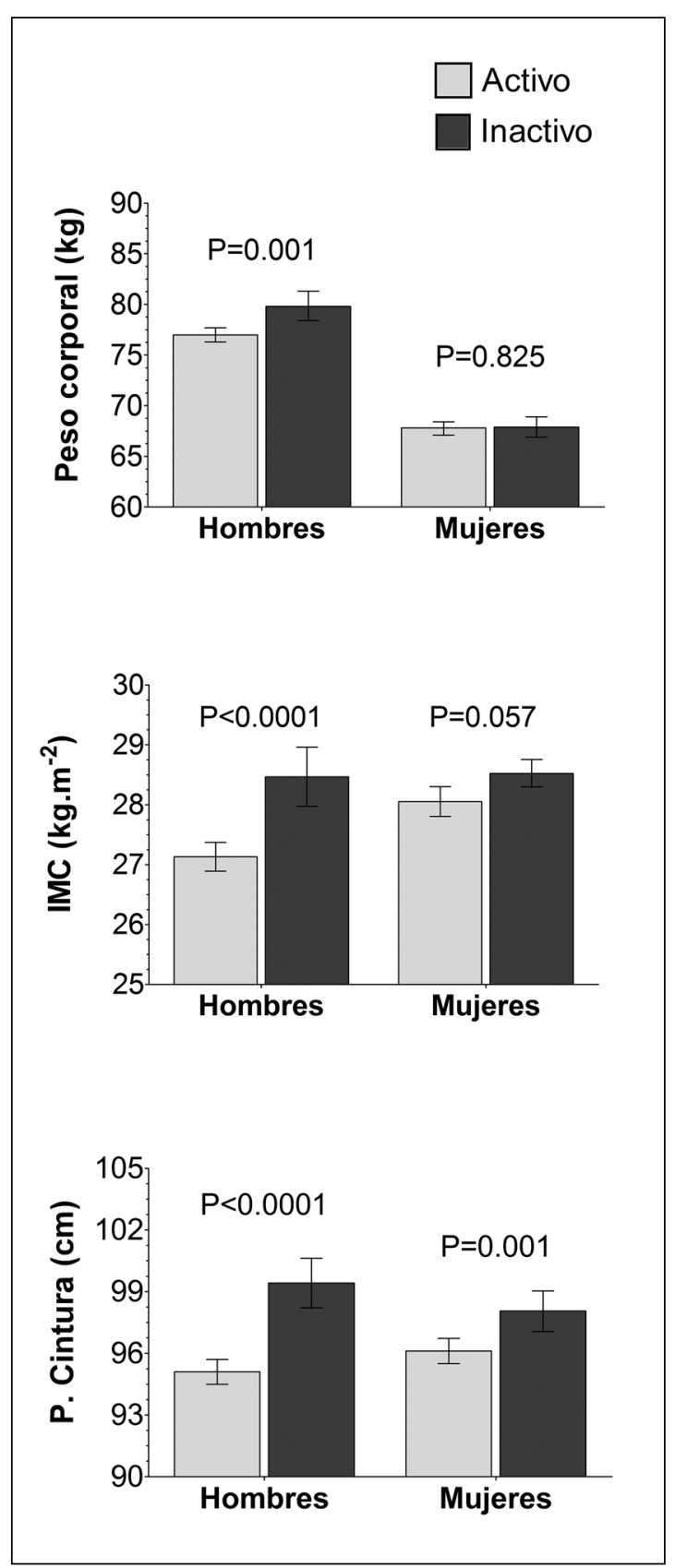

Figura 2. Variables antropométricas según niveles de actividad física y sexo, en población chilena. Los datos son presentados como promedio y su respectivo (95\% IC). El grupo "Activo" considera a aquellos individuos que cumplen con las recomendaciones de actividad física y realizan $>600$ $\mathrm{METs} / \mathrm{min} / \mathrm{semana}$ de actividad física y el grupo "Inactivo" incluye aquellos individuos que realizan < $600 \mathrm{METs} / \mathrm{min} /$ semana. Los análisis fueron ajustados por edad, educación, zona geográfica, tabaquismo, sedentarismo, consumo de sal, de frutas y verduras.

\section{Discusión}

El principal resultado de este estudio revela que ser físicamente inactivo se asocia a una salud cardiometabólica deteriorada, expresada en una mayor probabilidad de desarrollar obesidad, DMT2 e HTA tanto en hombres como en mujeres, mientras que solo se asocia a una mayor probabilidad de desarrollar obesidad central o síndrome metabólico en varones. Esta asociación fue independiente de los factores de confusión de tipo sociodemográficos, estilo de vida (tabaquismo, alimentación y sedentarismo) e IMC. Los resultados evidencian, además, que los efectos adversos de ser físicamente inactivo sobre estos parámetros de salud son mayores en hombres que en mujeres. Este último resultado podría estar relacionado por el factor protector adicional otorgado por los estrógenos quienes han sido ampliamente reconocidos sobre su impacto sobre la salud cardiovascular en mujeres antes de iniciar la menopausia ${ }^{20,21}$. Los resultado obtenidos podrían ser de utilidad para la definición de políticas en salud pública considerando que la ENS 2009-2010 reportó que 19,8\% de la población es físicamente inactiva, y que el costo económico asociado a la inactividad física en Chile, ha sido estimado en $\$ 103$ millones de dólares en el año $2013^{4}$. En consecuencia, estos resultados podrían ayudar a fortalecer la toma de decisiones en relación con la implementación de intervenciones y políticas públicas que tengan como objetivo incrementar los niveles de AF de la población.

Los hallazgos concuerdan con estudios previos, que reportan una relación inversa entre actividad física y obesidad, DMT2, HTA y síndrome metabólico tanto en Chile como en otros países a nivel mundial ${ }^{5,22-25}$. La promoción de la realización de AF como una medida de promoción y prevención primaria, secundaria y terciaria al desarrollo de distintas enfermedades no es un concepto nuevo, ya que desde tiempo antiguos ha sido fomentada: desde Hipócrates hasta actualmente la $\mathrm{OMS}^{26}$. Recientemente el grupo PURE (Prospective Urban Rural Epidemiology), evidenció que, tras el seguimiento de 7 años en 17 países de altos, medianos y bajos ingresos, $750 \mathrm{~min}$ de AF a la semana permitirían alcanzar los mayores beneficios en relación a la reducción de la mortalidad y las enfermedades cardiovasculares ${ }^{27}$. Si bien mayores 


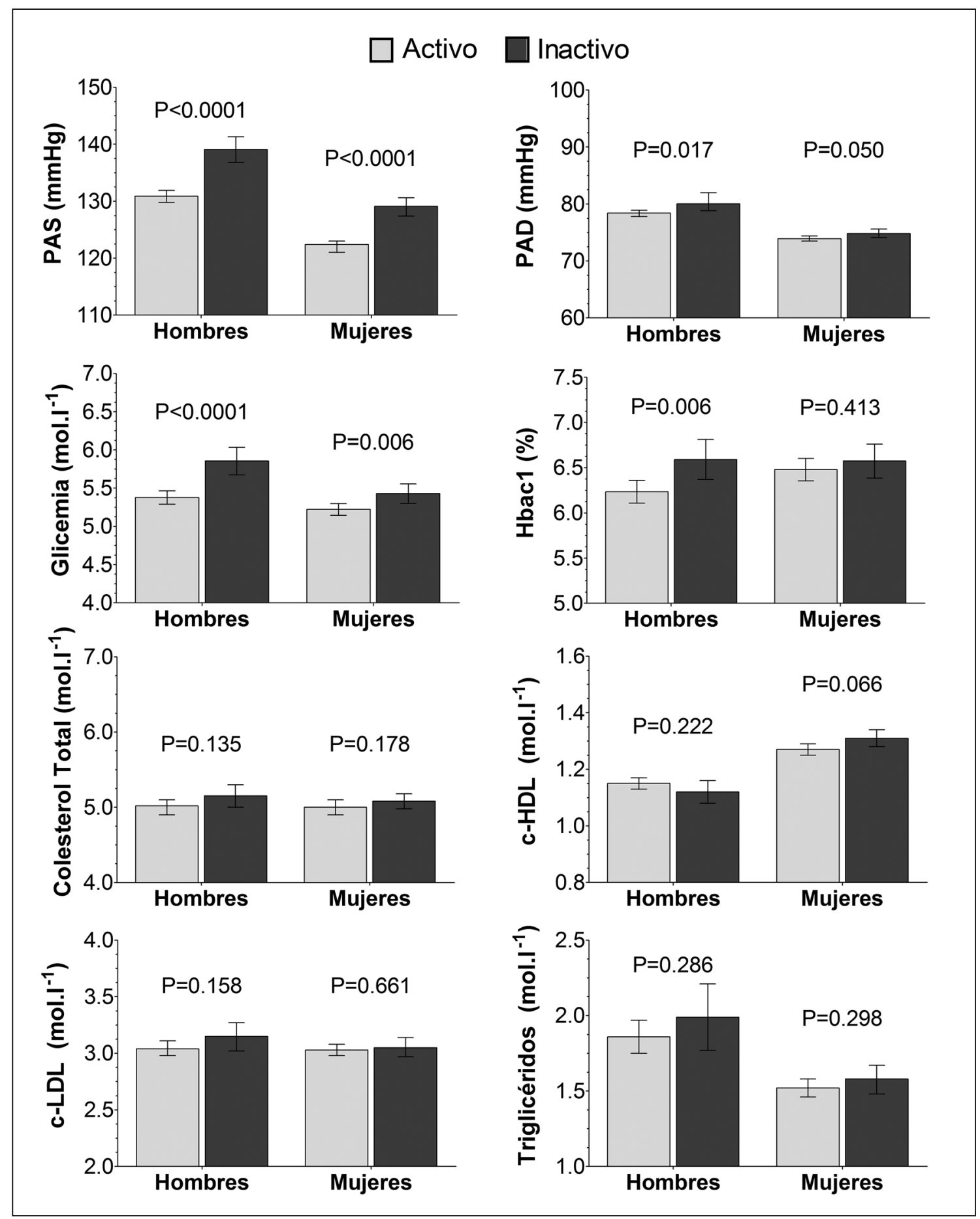

Figura 3. Marcadores de salud metabólica según niveles de actividad física y sexo, en población chilena. Los datos son presentados como promedio y su respectivo (95\% IC). El grupo "Activo" considera a aquellos individuos que cumplen con las recomendaciones de actividad física y realizan > $600 \mathrm{METs} / \mathrm{min} / \mathrm{semana}$ de actividad física y el grupo "Inactivo" incluye aquellos individuos que realizan < $600 \mathrm{METs} / \mathrm{min} / \mathrm{semana}$. Los análisis fueron ajustados por edad, educación, zona geográfica, tabaquismo, sedentarismo, consumo de sal, frutas y verduras e IMC. 

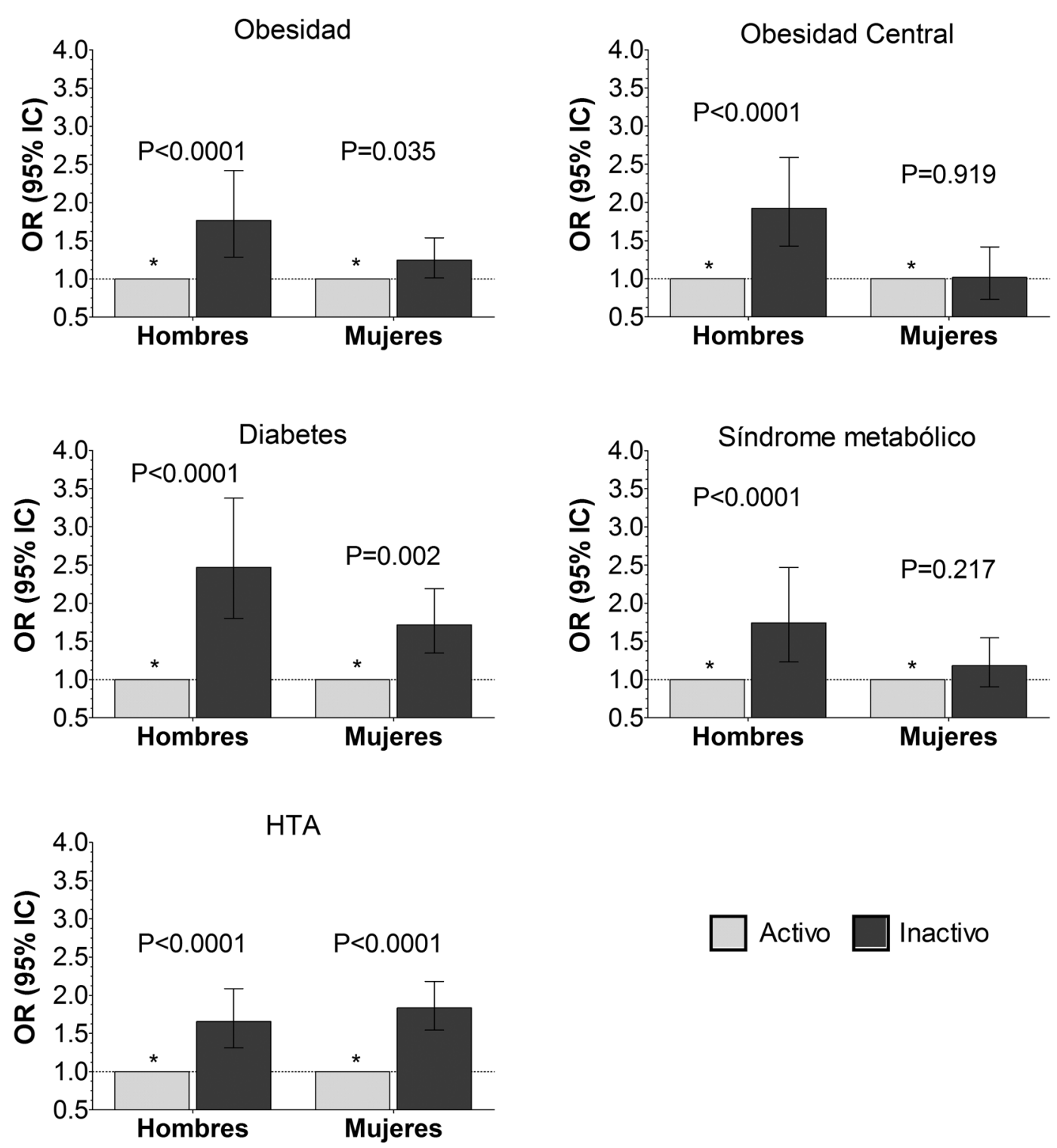

Activo Inactivo

Figura 4. Asociación de la inactividad física con obesidad, DMT2, HTA y síndrome metabólico según sexo en población chilena. Los datos son presentados como Odds ratio (OR) y su respectivo (95\% IC). El grupo "Activo" considera a aquellos individuos que cumplen con las recomendaciones de actividad física y realizan > $600 \mathrm{METs} / \mathrm{min} / \mathrm{semana}$ de actividad física y el grupo "Inactivo" incluye aquellos individuos que realizan < $600 \mathrm{METs} / \mathrm{min} / \mathrm{semana}$. Los análisis fueron ajustados por edad, educación, zona geográfica, tabaquismo, sedentarismo, consumo de sal, frutas y verduras e IMC.

niveles de AF se asocian a un perfil metabólico más favorable $\mathrm{e}^{1,5,23,27}$, los resultados muestran que con sólo cumplir la recomendación de AF y realizar al menos 150 min de AF de intensidad moderada a la semana, pueden observarse importantes reduc- ciones en la probabilidad de desarollar obesidad, DMT2, HTA y síndrome metabólico.

Una de las fortalezas de este estudio es la representatividad nacional de la ENS 2009-2010, como también la medición mediante protocolos estan- 
darizados de marcadores metabólicos. Se consideró una limitante de este estudio la medición de $\mathrm{AF}$ a través de cuestionarios auto-reportados. Si bien GPAQ ha sido validado internacionalmente ${ }^{28,29}$, existe evidencia que la medición de AF a través de cuestionarios genera una sobre estimación de los niveles reales de AF de la población ${ }^{7}$, lo cual podría ocultar la verdadera asociación entre AF y factores de riesgo cardiovascular tales como obesidad, DMT2, hipertensión y síndrome metabólico. Se sugiere que futuras encuestas o evaluaciones nacionales debiesen incorporar la medición objetiva de AF, usando por ejemplo acelerómetros de movimiento, que ya han sido utilizados en población chilena ${ }^{6,7}$. A pesar de que la ENS 2009-2010 carece de un diseño de seguimiento longitudinal o de estudio de control randomizado, por lo que no permite establecer una relación de causa-efecto; las asociaciones observadas en este trabajo han sido también reportadas en estudios longitudinales $s^{23,30-32}$. Otra limitante que es importante destacar es la falta de validación de un punto de corte para definir obesidad central en población chilena. Si bien, la Encuesta Nacional de Salud ${ }^{11}$ y la Guía de enfoque de riesgo para la prevención de enfermedades cardiovasculares en Chile ${ }^{17}$ sugieren diferentes puntos de corte ninguno de estos ha sido validado correctamente en población chilena. Este estudio utilizo el punto de corte de $\geq 90 \mathrm{o} \geq 80 \mathrm{~cm}$ para definición de obesidad central en hombres y mujeres, respectivamente. Lo cual podría explicar la no asociación de inactividad física con obesidad central o síndrome metabólico, ya que los niveles de corte para estas condiciones podrían no ser adecuados para población chilena. Cuando los análisis fueron realizados utilizando puntos de corte internacionales ( $>102 \mathrm{~cm}$ para hombres y $>88 \mathrm{~cm}$ para mujeres) inactividad física se asoció significativamente a obesidad central y síndrome metabólico (datos no han sido incluidos en este manuscrito).

En conclusión, los resultados de este estudio corroborar que tal como se ha identificado en otras poblaciones, la inactividad física se asocia a un aumento en la probabilidad de ser obeso, diabético, hipertenso en población chilena. Ser físicamente inactivo produce un deterioro en la salud cardiometabólica, en consecuencia, cumplir con las recomendaciones mínimas de al menos 150 min de AF moderada a la semana es esencial para el control y prevención de estos factores de riesgo. Estos resultados son relevantes en el ámbito de la salud pública, pudiendo servir como base para la implementación de políticas orientadas a la promoción de estilos de vida saludable en la población chilena desde tempranas edades.

Agradecimientos: Se agradece de manera especial a todos los participantes de la ENS 2009-10, al equipo profesional de la Escuela de Salud Pública, de la Facultad de Medicina de la Pontificia Universidad Católica de Chile, quienes desarrollaron y aplicaron la Encuesta Nacional de Salud y al Ministerio de Salud del Gobierno de Chile.

FP y CCM concibieron la pregunta de investigación. CCM, FP y XD realizaron los análisis estadísticos. CCM, FP y XD escribieron el manuscrito. Todos los autores revisaron críticamente el manuscrito y están de acuerdo con su versión final.

\section{Referencias}

1. Lee IM, Shiroma EJ, Lobelo F, Puska P, Blair SN, Katzmarzyk PT, et al. Effect of physical inactivity on major non-communicable diseases worldwide: an analysis of burden of disease and life expectancy. Lancet 2012; 380 (9838): 219-29.

2. WHO. Global health risks: mortality and burden of disease attributable to selected major risks. WHO, 2009.

3. Celis-Morales C, Lyall DM, Anderson J, Pell JP, Sattar N, Gill J. The association between physical activity and risk of mortality is modulated by grip strength and cardiorespiratory fitness: evidence from 498,135 UK-Biobank participants. European Heart Journal. 2016.

4. Ding D, Lawson KD, Kolbe-Alexander TL, Finkelstein EA, Katzmarzyk PT, van Mechelen W, et al. The economic burden of physical inactivity: a global analysis of major non-communicable diseases. The Lancet 2016; 388 (10051): 1311-24.

5. Celis-Morales C, Salas C, Alvarez C, Farías NA, Campillos RR, Leppe J, et al. Higher physical activity levels are associated with lower prevalence of cardiovascular risk factors in Chile. Rev Med Chile 2015; 143 (11): 1435-43.

6. Celis-Morales CA, Perez-Bravo F, Ibanes L, Sanzana R, Hormazabal E, Ulloa N, et al. Insulin Resistance in Chileans of European and Indigenous Descent: Evidence for an Ethnicity x Environment Interaction. Plos One 2011; $6(9)$.

7. Celis-Morales CA, Perez-Bravo F, Ibanez L, Salas C, Bailey ME, Gill JM. Objective vs. self-reported physical 
activity and sedentary time: effects of measurement method on relationships with risk biomarkers. PloS one 2012; 7 (5): e36345.

8. Gill JMR, Celis-Morales CA, Ghouri N. Physical activity, ethnicity and cardio-metabolic health: Does one size fit all? Atherosclerosis 2014; 232 (2): 319-33.

9. Hallal PC, Andersen LB, Bull FC, Guthold R, Haskell W, Ekelund U. Global physical activity levels: surveillance progress, pitfalls, and prospects. Lancet 2012; 380 (9838): 247-57.

10. WHO. Global recommendations on physical activity for health. World Health Organization, 2010.

11. MINSAL. Encuesta Nacional de Salud 2009-2010. Chile: Ministerio de Salud, 2010.

12. Celis-Morales C, Salas C, Alduhishy A, Sanzana R, Martínez M, Leiva A, et al. Socio-demographic patterns of physical activity and sedentary behaviour in Chile: results from the National Health Survey 2009-2010. Journal of Public Health 2015: 1-8.

13. Gill JMR, Malkova D. Physical activity, fitness and cardiovascular disease risk in adults: interactions with insulin resistance and obesity. Clinical science (London, England : 1979) 2006; 110 (4): 409-25.

14. Celis-Morales CA, Lyall DM, Anderson J, Iliodromiti S, Fan Y, Ntuk UE, et al. The association between physical activity and risk of mortality is modulated by grip strength and cardiorespiratory fitness: evidence from 498135 UK-Biobank participants. European Heart Journal 2017; 38 (2): 116-22.

15. Celis-Morales CA, Lyall DM, Welsh P, Anderson J, Steell L, Guo Y, et al. Association between active commuting and incident cardiovascular disease, cancer, and mortality: prospective cohort study. Bmj-British Medical Journal 2017; 357.

16. Celis-Morales CA, Petermann F, Hui L, Lyall DM, Iliodromiti S, McLaren J, et al. Associations Between Diabetes and Both Cardiovascular Disease and All-Cause Mortality Are Modified by Grip Strength: Evidence From UK Biobank, a Prospective Population-Based Cohort Study. Diabetes Care 2017; 40 (12): 1710-8.

17. MINSAL. Implementación del enfoque de riesgo en el Programa de Salud Cardiovascular. In: Gobierno de Chile MdS, editor. Santiago, Chile: Gobierno de Chile; 2014.

18. Alberti KGMM, Eckel RH, Grundy SM, Zimmet PZ, Cleeman JI, Donato KA, et al. Harmonizing the Metabolic Syndrome A Joint Interim Statement of the International Diabetes Federation Task Force on Epidemiology and Prevention; National Heart, Lung, and Blood Institute; American Heart Association; World Heart Federation; International Atherosclerosis Society; and International Association for the Study of Obesity. Circulation 2009; 120 (16): 1640-5.

19. WHO. Global Physical Activity Questionnaire: GPAQ version 2.0. World Health Organization, 2009.

20. Knowlton AA. Estrogen and the Female Heart 2014; 389 (1-2): 31-9.

21. Korzick DH, Lancaster TS. Age-Related Differences in Cardiac Ischemia-Reperfusion Injury: Effects of Estrogen Deficiency. Pflugers Archiv: European journal of physiology 2013; 465 (5): 669-85.

22. Manson JE, Greenland P, LaCroix AZ, Stefanick ML, Mouton CP, Oberman A, et al. Walking compared with vigorous exercise for the prevention of cardiovascular events in women. New England Journal of Medicine 2002; 347 (10): 716-25.

23. Shiroma EJ, Lee IM. Physical Activity and Cardiovascular Health Lessons Learned From Epidemiological Studies Across Age, Gender, and Race/Ethnicity. Circulation 2010; 122 (7): 743-52.

24. Saevereid HAS, Schnohr PS, Prescott EP. Speed and duration of walking and other leisure time physical activity and the risk of heart failure: the Copenhagen City Heart study. European Heart Journal 2013; 34: 658.

25. Nocon M, Hiemann T, Mueller-Riemenschneider F, Thalau F, Roll S, Willich SN. Association of physical activity with all-cause and cardiovascular mortality: a systematic review and meta-analysis. European Journal of Cardiovascular Prevention \& Rehabilitation 2008; 15 (3): 239-46.

26. Booth FW, Roberts CK, Laye MJ. Lack of exercise is a major cause of chronic diseases. Comprehensive Physiology 2012; 2 (2): 1143-211.

27. Lear SA, Hu W, Rangarajan S, Gasevic D, Leong D, Iqbal $\mathrm{R}$, et al. The effect of physical activity on mortality and cardiovascular disease in 130000 people from 17 high-income, middle-income, and low-income countries: the PURE study. Lancet (London, England) 2017; 390 (10113): 2643-54.

28. Bull FC, Maslin TS, Armstrong T. Global Physical Activity Questionnaire (GPAQ): Nine Country Reliability and Validity Study. Journal of Physical Activity \& Health 2009; 6 (6): 790-804.

29. Hoos T, Espinoza N, Marshall S, Arredondo EM. Validity of the Global Physical Activity Questionnaire (GPAQ) in Adult Latinas. Journal of Physical Activity \& Health 2012; 9 (5): 698-705.

30. Ahmed HM, Blaha MJ, Nasir K, Rivera JJ, Blumenthal RS. Effects of physical activity on cardiovascular disease. The American journal of cardiology 2012; 109 (2): 28895.

31. Balducci S, Zanuso S, Cardelli P, Salvi L, Bazuro A, 
Pugliese L, et al. Effect of High- versus Low- Intensity Supervised Aerobic and Resistance Training on Modifiable Cardiovascular Risk Factors in Type 2 Diabetes; The Italian Diabetes and Exercise Study (IDES). PloS one 2012; 7 (11).
32. Chomistek AK, Manson JE, Stefanick ML, Lu B, Sands-Lincoln M, Going SB, et al. Relationship of Sedentary Behavior and Physical Activity to Incident Cardiovascular Disease. Journal of the American College of Cardiology 2013; 61 (23): 2346-54. 